\title{
Situação de saúde da criança em área da região sul do Brasil, 1980-1992: tendências temporais e distribuição espacial*
}

\author{
The health situation of children in area of southern Brazil, 1980-1992: tendencies \\ over time and geographical distribution
}

\author{
Cesar G. Victora ${ }^{\star \star}$, Paulo Recena Grassi ${ }^{\star \star *}$, Angela Maria Schmidt***
}

\begin{abstract}
VICTORA, C.G. et al. Situação de saúde da criança em área da região sul do Brasil, 1980-1992: tendências temporais e distribuicão espacial. Rev. Saúde Pública, 28: 423-32 1994. Embora as estatísticas vitais sejam de fundamental importância para o planejamento e aval iação das ações de saúde, são poucos os Estados brasileiros que dispõem de sistema de registro com cobertura e agilidade suficiente para atingir estas metas. Objetivou, portanto, analisar os dados gerados no Rio Grande do Sul, Brasil, para descrever tendências temporais e distribuição espacial de indicadores de saúde infantil, incluindo os coeficientes de mortalidade infantil e de mortalidade proporcional de menores de um ano, prevalência de baixo peso ao nascer, e cobertura vacinal. Entre 1980 e 1992, observaram-se reduções marcantes na mortalidade infantil (de 39,0 para 19,3 por mil) e na mortalidade proporcional de menores de um ano (de $13,9 \%$ para 5,9\%). A prevalência de baixo peso ao nascer mostrou-se estável entre 8 e $10 \%$, tendo mesmo sido observado discreto aumento até 1991 . A cobertura de vacina tríplice oscilou marcadamente de ano a ano, entre $79 \%$ e $99 \%$. Houve forte correlação, ao nível de Delegacias Regionais de Saúde, entre mortalidade infantil e baixo peso ao nascer. Os 4 indicadores estudados foram combinados de forma a construir um escore para identificar as Delegacias de Saúde com maiores necessidades de intervenções sanitárias. A região sul do Estado, caracterizada pela presença de grandes latifúndios, mostrou os piores índices de saúde infantil.
\end{abstract}

Descritores: Mortalidade infantil, tendências. Baixo peso ao nascer. Registro de estatísticas vitais.

\section{Introdução}

O uso adequado das estatísticas vitais é de extrema importância para o planejamento e avaliação das ações de saúde. Infelizmente, são poucos os Estados brasileiros que dispõem de um sistema de registro com cobertura e agilidade suficiente para atingir estas metas. A presente análise utiliza os dados gerados no Rio Grande do Sul, tendo como objetivos: a) descrever a evolução temporal de alguns indicadores da saúde das crianças gaúchas no período de 1980 a 1992; b) analisar a distribuição espacial desses indicadores ao redor de 1990. Os dados sobre a tendência

* Estudo parcialmente financiado pelo PIMES (Programa Integrado de Melhoria Social) da Secretaria da Justiça, Governo do Estado do Rio Grande do Sul.

** Departamento de Medicina Social da Universidade Federal de Pelotas - Pelotas, RS - Brasil

*** Núcleo de Estatística, Divisão de Informação em Saúde, Secretaria da Saúde e Meio Ambiente do Estado do Rio Grande do Sul - Porto Alegre, RS - Brasil

Separatas/Reprints: C. C. Victora - Caixa Postal 464 96001-970 -Pelotas, RS - Brasil temporal e a distribuição dos indicadores de saúde infantil poderão ser utilizados como subsídio para o planejamento de ações sociais que visem a priorizar as crianças vivendo em regiões mais carentes do Estado. Os indicadores a serem utilizados estão descritos a seguir.

O primeiro indicador estudado, a mortalidade infantil, consiste tradicionalmente em um dos melhores índices não apenas da saúde infantil mas também do grau de desenvolvimento socioeconômico da comunidade ${ }^{1}$. Expresso como o número de óbitos por mil crianças nascidas vivas, o Coeficiente de Mortalidade Infantil (CMI) é costumeiramente dividido em dois subgrupos: mortalidade neonatal (6bitos do nascimento aos 27 dias) e pós-neonatal ou infantil tardia (dos 28 aos 365 dias de vida).

A mortalidade neonatal é causada primariamente pelos 6 bitos devidos a causas perinatais como prematuridade, problemas relacionados ao parto e malformações congênitas. Já a mortalidade pós-neonatal é causada basicamente por doenças infecciosas. Devido a estas etiologias, a mortalidade neonatal é de mais difícil prevenção do que a pós-neonatal, a qual pode ser evitada por intervenções específicas como imunizações 
e tratamento da diarréia e pneumonia, por exemplo, além de ser também sensível a melhorias na situação de alimentação, de saneamento e nas condições socioeconômicas mais amplas.

Recentemente, tem-se observado uma dissociação entre a queda da mortalidade infantil e as condições socieconômicas. Por exemplo, estudos descrevem importantes reduções na mortalidade em São Paulo e no Estado do Ceará desacompanhados de melhorias na situação socieconômica, sendo provável que essas reduções tenham sido devidas a intervenções específicas na área da medicina preventiva e curativa. Isto não invalida o uso da mortalidade infantil para a comparação de distintas áreas geográficas, mas alerta para o fato de que tendências decrescentes não indicam necessariamente melhoria nas condições de vida da população.

A mortalidade pré-escolar, por outro lado, refere-se a faixa etária de um a cinco anos incompletos, sendo expressa como o número de óbitos por cada mil crianças, nesta faixa etária. Na grande maioria das populações, a mortalidade pré-escolar é várias vezes menor do que a mortalidade infantil por estarem as crianças mais velhas já livres dos problemas perinatais, com um sistema imunológico completamente desenvolvido e, inclusive, por haverem as crianças mais suscetíveis já morrido durante o primeiro ano de vida.

Outro indicador de grande valor é o percentual de baixo peso ao nascer, definido como um peso inferior a $2.500 \mathrm{~g}$. Este é, isoladamente, o principal determinante proximal da mortalidade infantil ${ }^{y}$. Existem dois principais mecanismos que levam ao baixo peso ao nascer: a prematuridade e o retardo de crescimento intra-uterino. A prematuridade ocorre quando a criança nasce antes de completar 37 semanas de gestação. Já o retardo de crescimento intra-uterino, também conhecido como desnutrição fetal, ocorre quando a criança nasce com peso abaixo do valor-limite para sua idade gestacional. A maior parte destas últimas crianças são nascidas a termo. Entre as principais causas de baixo peso ao nascer encontram-se as infecções, a desnutrição materna e o fumo.

$O$ peso ao nascer, como a mortalidade infantil, constitui também um excelente indicador socioeconômico. Na verdade, tem sido proposto ${ }^{6}$ que este indicador seria mesmo superior à mortalidade infantil, por ser menos influenciável do que esta a intervenções médico-sanitárias específicas.

Em termos de indicadores amplamente disponíveis de cobertura de serviços preventivos, a cobertura vacinal ocupa lugar de destaque. As vacinas constituem uma proteção essencial para o desenvolvimento sadio na infância. Escolheuse como indicador, na presente análise, o percentual de crianças menores de um ano que haviam recebido as três doses recomendadas da vacina tríplice (contra difteria, coqueluche e tétano). Esta é a vacina que exige maior organização dos serviços de saúde por consistir de três doses injetáveis, aos dois, quatro e seis meses de vida.

Tentou-se ainda obter dados sobre séries temporais ou distribuições geográficas de diversos outros indicadores de saúde e nutrição. Entre estes incluem-se dados de altura de escolares (um indicador nutricional), de número de hospitalizações de crianças conforme a causa, e de prevalências de desnutrição por região. Além do peso ao nascer, já discutido acima, o único outro indicador nutricional disponível foi o resultado da Pesquisa Nacional sobre Saúde e Nutrição, realizada em $1989^{3,4}$. Na presente pesquisa, $8,2 \%$ das crianças gaúchas apresentaram déficit de altura/idade. A prevalência de desnutrição para todo o país foi estimada em $15,9 \%$, variando de 4,9\% em Santa Catarina a 33,8\% no Maranhão e Piauí. Além de Santa Catarina, os Estados de São Paulo e Mato Grosso do Sul apresentaram menores freqüências do que o Rio Grande do Sul. Não foi possível estratificar este resultado para as diferentes regiões do Estado, devido ao reduzido tamanho da amostra estudada.

Para entender-se as inter-relações entre os indicadores propostos, partiu-se do pressuposto de que as condições materiais de existência, determinadas pela formação social, econômica e política de uma comunidade influenciam os padrões de saúde e nutrição ${ }^{7}$. Esta influência se dá através de fatores intermediários como água e saneamento, disponibilidade de alimentos, poder aquisitivo da família, condições de moradia, entre outros.

Assim, espera-se que em regiōes de intensas desigualdades sociais e de baixo poder aquisitivo da população encontrem-se maiores níveis de mortalidade e de desnutrição. No caso do Rio Grande do Sul, pesquisa realizada com dados de $1980^{8}$, mostrou que além do poder aquisitivo a questão da propriedade da terra exercia forte influência sobre a morbi-mortalidade infantil. $O$ norte do Estado, caracterizado por minifúndios, apresentava em 1980 baixos índices de mortalidade e de desnutrição, enquanto que a região sul, dos latifúndios, apresentava índices elevados.

A organização dos serviços de saúde, por outro lado, não leva em conta estas desigualdades sociais e médico-sanitárias. Os serviços são 
freqüentemente localizados em áreas onde menos são necessários, acentuando as desigualdades existentes.

Estes breves comentários sobre as relações entre os indicadores a serem analisados visaram a contribuir para o melhor entendimento das associacões mostradas no presente trabalho.

\section{Fonte dos Dados e Metodologia}

Foram obtidos junto à Secretaria de Saúde e do Meio Ambiente dados sobre mortalidade, peso ao nascer e cobertura vacinal. A divisão geográfica do Estado, utilizada por essa Secretaria, é a de Delegacias Regionais de Saúde (DRS). Esta divisão identifica 16 regiões geográficas, não sendo, no entanto, coincidente com a divisão em microrregiões homogêneas utilizada pela Fundação Instituto Brasileiro de Geografia e Estatística. Foi impossível analisar os dados conforme esta classificação devido à falta de disponibilidade de indicadores de saúde desagregados por município. Para alguns indicadores, como os dados de mortalidade, baixo peso ou cobertura vacinal para todo o Estado, foi possível obter dados até 1991 ou 1992. As análises geográficas, no entanto, foram realizadas com dados referentes aos triênios 1988-90 ou 1989-91.

\section{Mortalidade infantil e pré-escolar}

Os dados de óbitos registrados no Rio Grande do Sul, entre 1980 e 1992 foram obtidos da Secretaria da Saúde e do Meio Ambiente. Estes óbitos incluem apenas os óbitos registrados em cartório, e portanto podem ser afetados em maior ou menor grau pelo sub-registro, isto é, crianças que morrem e são enterradas sem os procedimentos legais. Embora se acredite que no Rio Grande do Sul o índice de sub-registro seja reduzido, pesquisa realizada em Pelotas, em 1982, mostrou que $24 \%$ dos óbitos infantis não haviam sido registrados ${ }^{7}$. No entanto, nova pesquisa realizada na mesma cidade em 1993 mostrou que o sub-registro havia praticamente desaparecido*.

Os dados obtidos incluíram o número total de óbitos infantis, o número de óbitos infantis por período (neonatal ou pós-neonatal) e por causas, e o número de óbitos pré-escolares. A mortalidade infantil por DRS foi também obtida.

Utilizando-se as populações estimadas de crianças menores de um ano e de um a quatro anos (ver abaixo), calcularam-se os coeficientes

* Dados inéditos de mortalidade infantil e pré-escolar, respectivamente, por mil crianças. Optou-se por utilizar o número de menores de um ano como denominador para a mortalidade infantil por acreditar-se ser este mais confiável do que o dado de nascidos vivos do registro civil.

Indicador freqüentemente utilizado para tentar contornar - pelo menos parcialmente - o problema do sub-registro, é a mortalidade proporcional, em que, por exemplo, o número de óbitos infantis é dividido pelo número total de óbitos em todas as idades. Is to parte do pressuposto de que se há omissão de óbitos isto ocorreria em todas as idades, e, portanto, altas proporções de óbitos infantis revelariam uma situação sanitária inadequada. Estes indicadores também foram calculados.

Entre os óbitos de crianças menores de um ano, calculou-se também o percentual de óbitos por alguns grupos de causas selecionadas, codificadas conforme a $9^{a}$ versão da Classificação Internacional de Doenças. Para fins de apresentação, as causas de morte foram classificadas como infecciosas (seção $I$ da CID), respiratória (seção 8), malformações (seção 14), perinatais ( seção 15) e outras causas (demais seções).

\section{Baixo peso ao nascer}

Dados sobre o peso ao nascer foram obtidos a partir do sistema de registro de nascimentos (SINASC) da Secretaria da Saúde e do Meio Ambiente. Estes dados estão disponíveis para os períodos de $1980-83$ e para $1989-92$, havendo o sistema de coleta sido interrompido de 1984 a 1988. Os dados se restringem a nascimentos hospitalares.

A cobertura do sistema - calculada pela divisão do número de nascimentos incluídos pelo total de nascimentos no Estado - era quase que total no início da década, quando atingiu 97\% (média de 1980 a 1983). Após a interrupção do sistema, a cobertura estimada caiu para $89 \%$ no triênio 1989-91.

O percentual de baixo peso ao nascer foi calculado dividindo-se o número de nascimentos com menos de 2.500 gramas pelo total de nascimentos notificados ao sistema, excluídos os nascimentos de peso ignorado.

\section{Cobertura vacinal}

A cobertura vacinal foi também obtida através de dados da Secretaria da Saúde e do Meio Ambiente, referentes ao número de crianças de menores de um ano que receberam a terceira 
dose da vacina tríplice, para todo o Estado e por DRS. Estes dados são notificados pelos Centros e Postos de Saúde, e pelas demais entidades que realizam a vacinação. A cobertura foi calculada dividindo-se este número pela população estimada de menores de um ano.

\section{População de crianças}

Dados do Censo Demográfico de 1980 forneceram o número de menores de um ano para todo o Estado e por DRS. Como os resultados do Censo Demográfico de 1991, discriminados por faixa etária, não estavam disponíveis por ocasião da análise, utilizou-se estimativa realizada pela Fundação de Economia e Estatística*, baseada na população geral de cada DRS multiplicada pela proporção estimada de menores de um ano na população. Cálculo análogo foi realizado para a faixa etária de 1 a 4 anos.

\section{Resultados}

\section{Tendências temporais}

As análises seguintes referem-se às tendências temporais observadas para os indicadores de saúde de 1980 a 1992.

\section{Mortalidade infantil e pré-escolar}

Durante a década de 1970 , a mortalidade infantil apresentou tendência decrescente no Estado, de 48 por mil em 1970 para 39,0 por mil em 1980, ou seja, uma redução de $19 \%$.

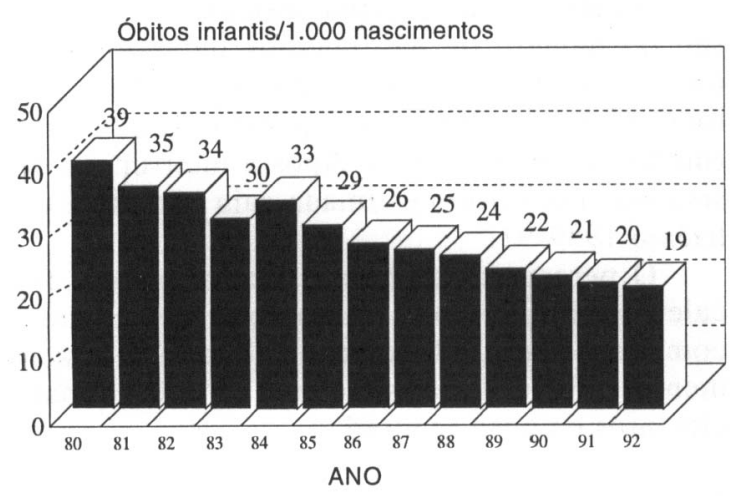

Figura 1. Coeficientes de mortalidade infantil. Rio Grande do Sul, 1980-1992.

Fonte: Secretaria de Saúde e Meio Ambiente. Fundaçăo de Economia e Estatística.

* Dados inéditos.

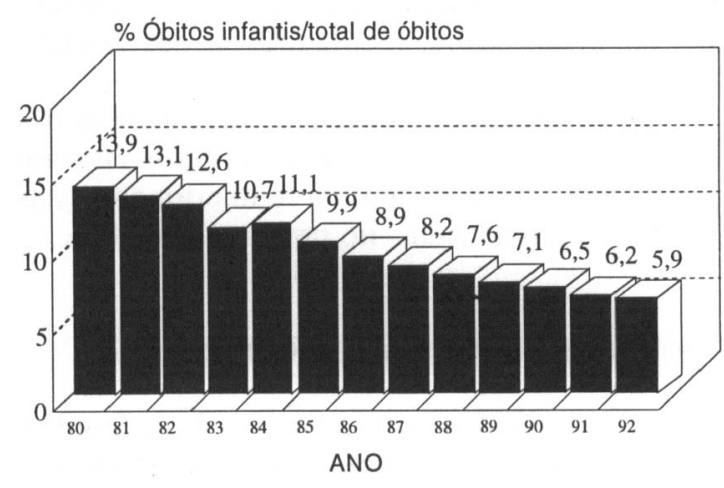

Figura 2. Mortalidade infantil proporcional. Rio Grande do Sul, 1980-1992.

Fonte: Secretaria de Saúde e Meio Ambiente. Fundação de Economia e Estatística.

A tendência à redução intensificou-se nos anos 80 (Fig. 1), atingindo o nível de 19,3 por mil em 1992, ou seja, um decréscimo de $50 \%$. Nesta década, a mortalidade geral (isto é, em todas as idades) manteve-se relativamente constante, sendo de 6,7 por mil pessoas em 1980 e de 6,2 por mil em $1990^{5}$.

Para verificar até que ponto esta queda poderia estar sendo afetada por alterações no registro de óbitos, calculou-se a mortalidade proporcional de menores de um ano, a qual como anteriormente comentado, é menos suscetível ao sub-registro do que o coeficiente de mortalidade infantil. Entre 1980 e 1992 , esta reduziu-se de $13,9 \%$ para $5,9 \%$ (Fig. 2), ou seja, um decréscimo de $58 \%$. Portanto, tudo indica que a mortalidade infantil tenha sido reduzida a cerca da metade durante a década.

Apesar deste declínio, o Rio Grande do Sul ainda apresenta mortalidade infantil de cerca de 19 por mil, bastante superior a países latinoamericanos como Cuba (11 por mil em 1990),

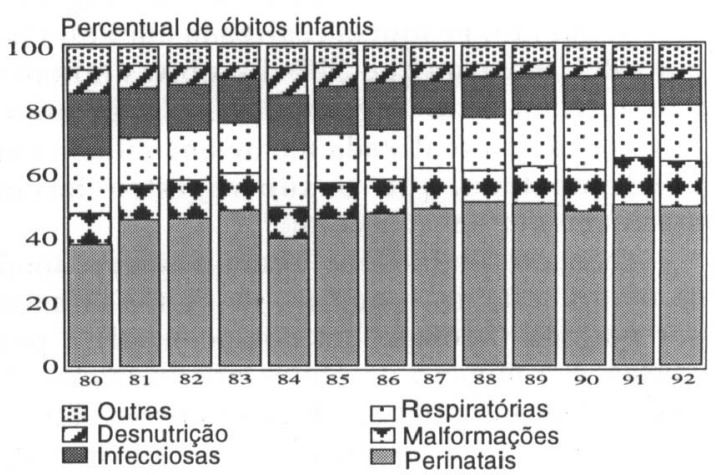

Figura 3. Mortalidade infantil proporcional por causas. Rio Grande do Sul, 1980-1992.

Fonte: Secretaria de Saúde e Meio Ambiente. Fundação de Economia e Estatística. 
sem falar em países desenvolvidos como o Japão ( 5 por mil), Suéciae Finlândia (ambos com 6 por mil) ${ }^{1}$.

Ao analisar-se o declínio na mortalidade conforme grupos de causas, observa-se que as causas infecciosas (incluindo a diarréia) e a desnutrição apresentaram reduções mais rápidas do que o conjunto da mortalidade (Fig. 3). As doenças respiratórias mantiveram o mesmo percentual, enquanto que as causas perinatais (prematuridade, problemas de parto) e as malformações aumentaram relativamente. Notese que mesmo este aumento relativo constitui uma redução, pois durante o período coberto por esta análise (Fig. 3) o coeficiente de mortalidade infantil reduziu-se marcadamente em $50 \%$.

A mortalidade pós-neonatal (ocorrida entre os 28 e 364 dias de vida) representava $49,6 \%$ dos óbitos infantis em 1980, decrescendo ligeiramente para 43,6\% em $1990^{5}$. Esta redução foi modesta, bastante inferior ao que seria esperado em face do importante declínio na mortalidade infantil, uma vez que normalmente a queda ocorre principalmente às custas dos óbitos mais tardios. Portanto, esperar-se-ia que um CMI de 20 por mil correspondesse a um percentual de óbitos pós-neonatais bastante inferior aos cerca de $40 \%$ observados. Em estudo realizado em Pelotas, em $1982^{7}$, esta proporção de $40 \%$ correspondeu a um CMI de 34 por mil (grupo de renda entre 1 e 3 salários mínimos daquele estudo). Quando o CMI foi de 16,5 por mil (grupo de renda superior a $3 \mathrm{SM}$ em nosso estudo), apenas $21 \%$ dos óbitos foram pósneonatais. Este resultado sugere a existência de sub-registro de óbitos no âmbito estadual.

Uma possível explicação para as reduções observadas é que intervenções específicas no primeiro ano de vida estejam resultando no "adiamento" de mortes, com os óbitos passando a

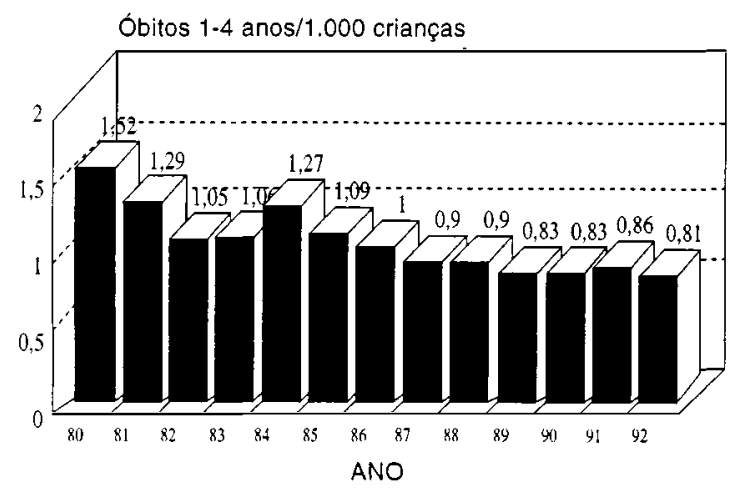

Figura 4. Coeficientes de mortalidade pré-escolar. Rio Grande do Sul, 1980-1992.

Fonte: Secretaria de Saúde e Meio Ambiente. Fundação de Economia e Estatística.

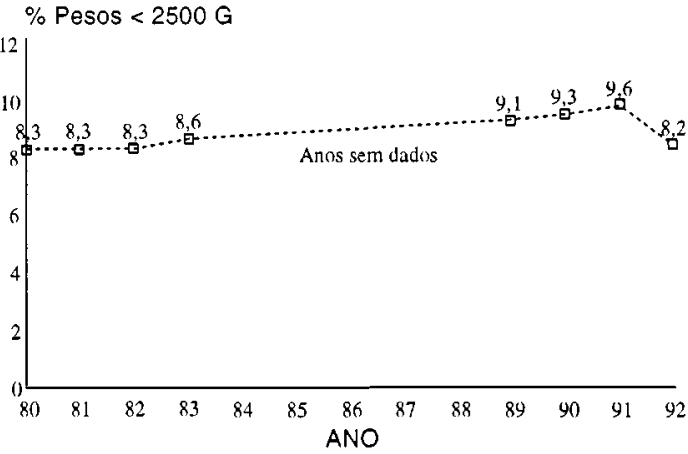

Figura 5. Índices de baixo peso ao nascer. Rio Grande do Sul, 1980-92.

Fonte: Secretaria de Saúde e Meio Ambiente. Fundação de Economia e Estatística.

ocorrer após o primeiro aniversário. Isto não aconteceu, pois a mortalidade pré-escolar (idades de 1 a 4 anos) também decresceu marcadamente na década, de 1,52, por 1000, para 0,81 (Fig. 4), uma redução de $47 \%$, ou seja, quase idêntica à redução na mortalidade infantil. Conforme normalmente ocorre, o risco de morte após o primeiro ano de vida é muitas vezes menor do que para os recém-nascidos ${ }^{1}$.

\section{Baixo peso ao nascer}

Dados sobre peso ao nascer estão disponíveis para os anos de 1980 a 1983 e de 1988 a 1992 (Fig. 5). Ao contrário dos dados de mortalidade, o percentual de baixo peso ao nascer não apenas deixaram de apresentar redução, mas aumentaram em $11,5 \%$ até 1991 . A brusca queda de $9,6 \%$, em 1991, para $8,2 \%$, em 1992 , no entanto, é difícil de interpretar.

É pouco provável que esta série histórica

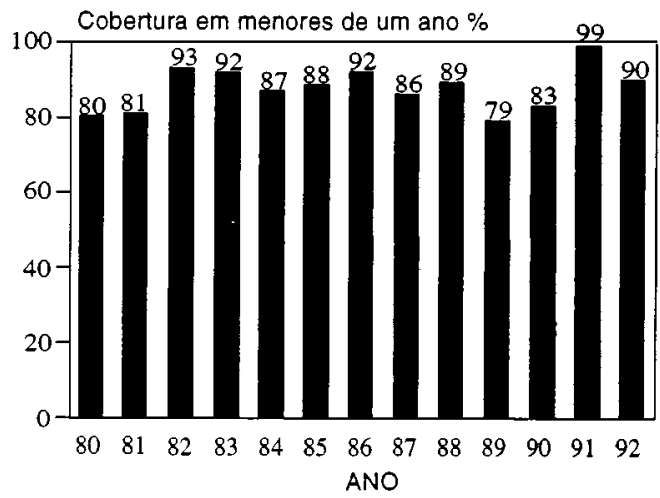

Figura 6. Cobertura vacinal (Tríplice). Rio grande do Sul, 1980-1992.

Fonte: Secretaria de Saúde e Meio Ambiente. Fundação de Economia e Estatística. 
tenha sido afetada pela mudança na cobertura estimada do sistema de nascimentos, de $97 \%$, em 1980-83, para $89 \%$, no triênio $1989-91$, e para $92 \%$, em 1992. Se a queda na cobertura ocorreu preferencialmente nas regióes mais carentes, esperar-se-ia que o sistema revelasse menor prevalência de baixo peso ao nascer no final da década, o que não ocorreu.

A estabilidade no percentual de crianças com baixo peso ao nascer sugere que as condições gerais das gestantes no tocante a estado nutricional e infecções, assim como do atendimento pré-natal, não tenham apresentado progresso na década. Como já mencionado existe proposta de que o baixo peso ao nascer seria um melhor indicador das condições subjacentes de saúde do que a mortalidade infantil, por ser esta última mais suscetível a intetvenções específicas da medicina preventiva e curativa ${ }^{6}$.

\section{Cobertura vacinal}

A Figura 6 mostra que a cobertuta de menores de um ano pela vacina tríplice oscilou substancialmente entre 1980 e 1991 . A cobertura oscilou entre $80 \%$ e $90 \%$ na década aumentando consideravelmente em 1991. Comparativamente a outros Estados brasileiros, os níveis são adequados mas são preocupantes as amplas oscilações observadas, que sugerem ser a cobertuta vacinal mais dependente de campanhas em anos específicos do que da atividade rotineira dos serviços de saúde.

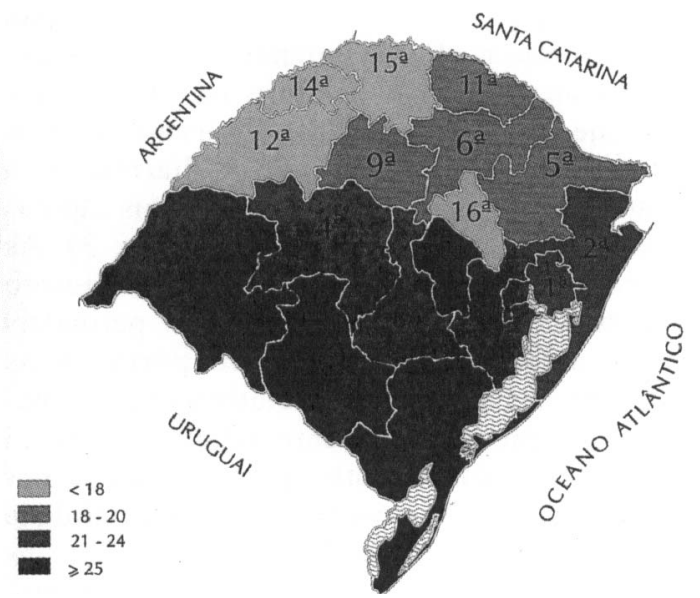

Figura 7. Coeficiente de mortalidade infantil por 1.000 . Rio Grande do Sul. Divisão em Delegacias Regionais de Saúde.

\section{Distribuição Geográfica}

A distribuição geográfica dos indicadores de saúde levou em conta a divisão do Estado em Delegacias Regionais de Saúde. Para facilitar a apresentação, somente os municípios-sede de cada Delegacia são mencionados no texto, ficando subentendido que o dado refere-se a toda a Delegacia.

Para evitar flutuações ocorridas de ano para ano, os dados apresentados (Tabela 1) referemse aos triênios 1988-90 ou 1989-91.

Tabela 1. Indicadores de saúde selecionados para as Delegacias Regionais de Saúde. Rio Grande do Sul.

\begin{tabular}{|c|c|c|c|c|}
\hline $\begin{array}{l}\text { Delegacia Regiona de } \\
\text { Saúde e Município-Sede }\end{array}$ & $\begin{array}{r}\text { Coeficiente de } \\
\text { mortalidade } \\
\text { infantil por } 1.000\end{array}$ & $\begin{array}{r}\text { Mortalidede } \\
\text { proporcional } \\
\text { em menores de } \\
\text { um ano }(\%)\end{array}$ & $\begin{array}{r}\text { Percentual de } \\
\text { baixo peso ao } \\
\text { nascer }\end{array}$ & $\begin{array}{r}\text { Cobertura de } \\
\text { menores de } \\
\text { um ano com } \\
\text { vacina tríplice }\end{array}$ \\
\hline Triênio & $1988-90$ & $1988-90$ & $1989-91$ & $1989-91$ \\
\hline$\overline{19 \text { (Porto Alegre) }}$ & 23,5 & 7,4 & 9,5 & 80,3 \\
\hline 2 (Porto Alegre) & 21,1 & 7,2 & 9,1 & 88,3 \\
\hline $3^{a}$ (Pelotas) & 31,4 & 7,2 & 10,4 & 80,4 \\
\hline $\begin{array}{l}4^{\text {a }} \text { (Santa Maria) } \\
5^{\text {a }} \text { (Caxias do Sul) }\end{array}$ & $\begin{array}{l}21,7 \\
20,1\end{array}$ & $\begin{array}{l}6,0 \\
6,5\end{array}$ & $\begin{array}{r}10,1 \\
8,8\end{array}$ & $\begin{array}{r}90,7 \\
100,0\end{array}$ \\
\hline 6 (Passo Fundo) & 20,1 & 7,1 & 9,2 & 91,1 \\
\hline $7^{a}$ (Bagé) & 30,5 & 7,4 & 10,4 & 81,2 \\
\hline $8^{a}$ (Cachoeira do Sul) & 22,2 & 6,2 & 8,9 & 84,5 \\
\hline $9^{a}$ (Cruz Alta) & 19,0 & 6,4 & 10,1 & 96,9 \\
\hline $10^{\mathrm{a}}$ (Alegrete) & 33,8 & 10,0 & 10,2 & 83,3 \\
\hline $11^{a}$ (Erechim) & 20,1 & 8,0 & 8,0 & 94,0 \\
\hline $12^{a}$ (Santo Ângelo) & 17,3 & 6,8 & 9,2 & 86,0 \\
\hline 13 ${ }^{\text {a }}$ (Santa Cruz do Sul) & 25,6 & 6,6 & 9,5 & 87,2 \\
\hline 14" (Santa Rosa) & 15,8 & 5,8 & 8,1 & 93,3 \\
\hline 15a (Palmeira das Missōes) & 13,4 & 6,5 & 8,0 & 84,0 \\
\hline $16^{\mathrm{a}}$ (Lajeado) & 16,9 & 4,9 & 7,4 & 92,8 \\
\hline
\end{tabular}




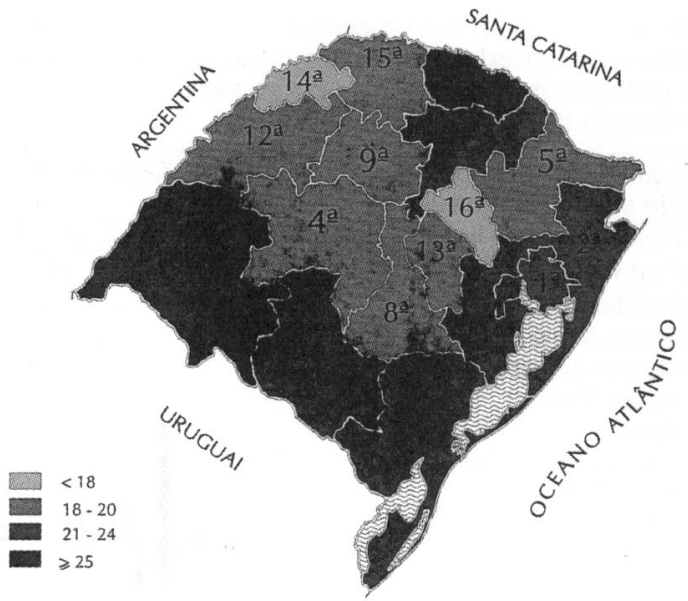

Figura 8. Mortalidade proporcional em menores de 1 ano, 1988-1990. Rio Grande do Sul. Divisāo em Delegacias Regionais de Saúde.

\section{Mortalidade infantil}

$O$ coeficiente do mortalidade infantil variou do 13,4 por mil (DRS Palmeira das Missões) a 33,8 por mil (Alegrete). Baixas mortalidades foram observadas também em Lajeado, Santo Ângelo, Cruz Alta e Santa Rosa, enquanto que índices elevados também foram notados em Pelotas e Bagé. Observase, portanto, nítido gradiente de mortalidade infantil com os índices altos no sul e baixos no norte do Estado (Fig. 7).

A mortalidade proporcional de menores do um ano apresentou uma distribuição razoavelmente similar a do coeficiente de mortalidade infantil, variando de $4,9 \%$ em Lajeado,

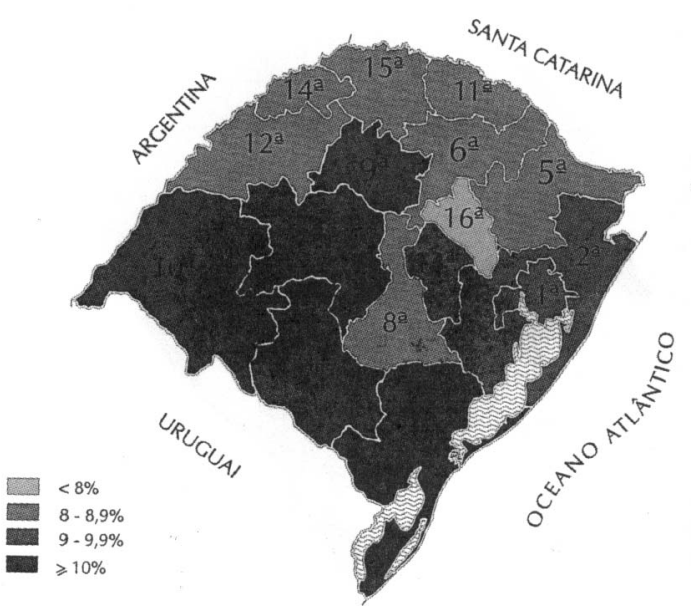

Figura 9. Percentual de baixo peso ao nascer $(<2.500 \mathrm{~g})$, 1989-1991. Rio Grande do Sul. Divisāo em Delegacias Regionais de Saúde.

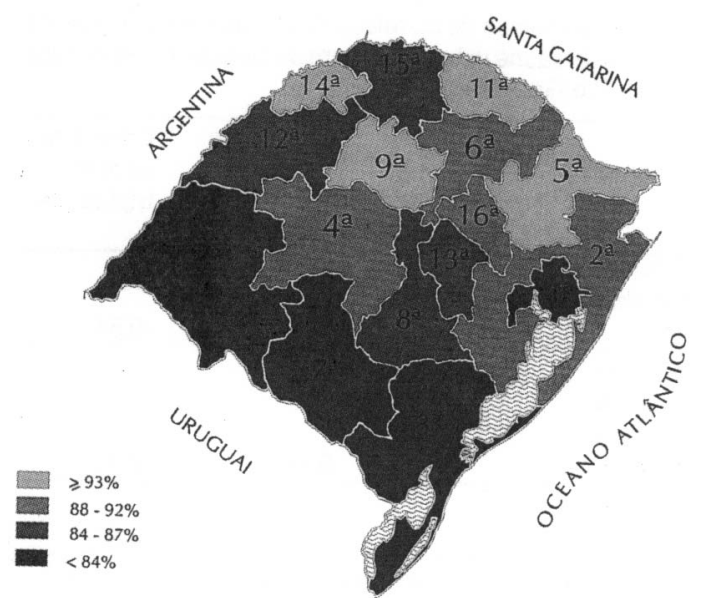

Figura 10. Cobertura vacinal, 1989-1991. Rio Grande do Sul. Divisāo em Delegacias Regionais de Saúde.

a 10,0\% em Alegrete. Novamente o gradiente norte-sul pode ser observado (Fig. 8), embora não tão claramente quanto para o coeficiente de mortalidade infantil.

A variacão geográfica na mortalidade préescolar não foi estudada, principalmente porque o reduzido número de óbitos produziu estimativas bastante instáveis.

\section{Baixo peso ao nascer}

Os percentuais de recém-nascidos com peso inferior a $2.500 \mathrm{~g}$ variaram de $7,4 \%$, em Lajeado, para $10,4 \%$, em Pelotas e Bagé. Índices superiores a $10 \%$ foram também encontrados em Cruz Alta, Alegrete e Santa Maria, enquanto que prevalências inferiores a $8 \%$ foram observadas também em Erechim e Palmeira das Missões. A Figura 9 mostra que uma clara divisião entre o norte e o sul do Estado pode ser novamente constatada.

Coef. mortalidade infantil por 1.000

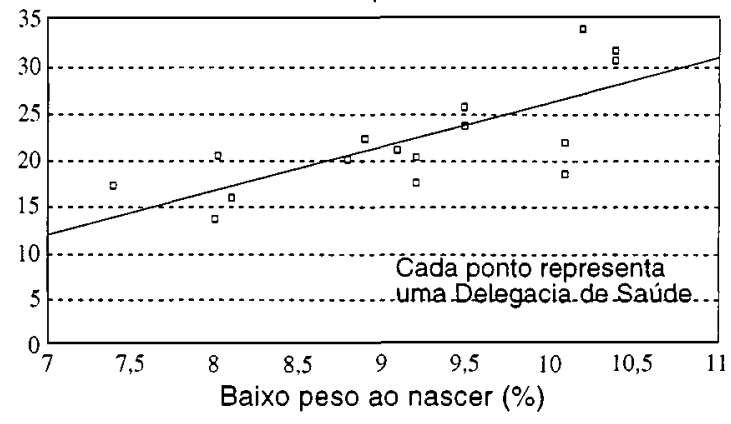

Figura 11. Relação entre a mortalidade infantil e o baixo peso ao nascer. Rio Grande do Sul, 1989-91.

Fonte: Secretaria de Saúde e Meio Ambiente. Fundação de Economia e Estatística. 
Tabela 2. Coeficientes de correlaçăo linear de Pearson entre os indicadores de saúde estudados, para as Delegacias Regionais de Saúde. Rio Grande do Sul.

\begin{tabular}{llll}
\hline Indicadores & $\begin{array}{l}\text { Mortalidade } \\
\text { proporcional } \\
\text { em menores } \\
\text { de um ano }\end{array}$ & $\begin{array}{l}\text { Baixo peso } \\
\text { ao nascer }\end{array}$ & $\begin{array}{l}\text { Cobertura } \\
\text { vacinal } \\
\text { tríplice }\end{array}$ \\
\hline $\begin{array}{l}\text { Coeficiente } \\
\text { de mortalidade } \\
\text { infantil }\end{array}$ & $0,66^{*}$ & $0,75^{*}$ & $-0,54$ \\
\hline
\end{tabular}

Mortalidade

proporcional

em menores

\begin{tabular}{lll} 
de um ano & 0,45 & $-0,38$ \\
\hline Baixo peso & &
\end{tabular}

ao nascer

* Correlação estatisticamente significante.

\section{Cobertura vacinal}

A distribuição geográfica da cobertura com vacina tríplice (Fig. 10) foi bem menos clara do que a dos indicadores anteriores. As piores coberturas - abaixo do 84\% - foram observadas em Porto Alegre, Pelotas, Bagé e Alegrete, estando as mais elevadas - $85 \%$ ou mais - em Lajeado, Santa Rosa, Erechim, Cruz Alta e Caxias do Sul.

\section{Análise de Correlação}

Para avaliar a magnitude das associações geográficas entre os quatro indicadores estudados, obteve-se uma matriz de correlações utilizando-se o coeficiente de Pearson, tendo-se como unidades de análise as 16 DRSs.

A Tabela 2 mostra que a mais forte correlação positiva $(0,75)$ ocorreu entre o coeficiente de mortalidade infantil e o baixo peso ao nascer, comprovando a forte associação entre estes dois fatores, ou seja, que em áreas de maior prevalência do baixo peso, a mortalidade é maior. A Figura 11 mostra graficamento esta associação.

A correlação entre o coeficiente de mortalidade infantil e a mortalidade proporcional de menores de um ano foi de moderada a forte $(0,66)$, mas um pouco abaixo do que se poderia esperar para duas variáveis que, em teoria, estariam medindo o mesmo fenômeno. Esta diferença pode ser devida a problemas metodológicos como o sub-registro ou erros na estimativa dos denominadores.

Já a associação entre o baixo peso ao nascer e a mortalidade proporcional foi de 0,45 , ou seja, moderada.

Em relação à cobertura vacinal, note-se que esta esteve inversamente associada com os demais indicadores, particularmente com o coeficiente
Tabela 3. Ordenação das Delegacias Regionais de Saúde, conforme os indicadores de saúde (o $1 \%$ lugar corresponde aos melhores indicadores $\theta$ o décimo sexto aos piores). Rio Grande do Sul.

\begin{tabular}{|c|c|}
\hline $\begin{array}{l}\text { Delegacia Regional de } \\
\text { Saúde e Município-Sede }\end{array}$ & Colocação \\
\hline$\overline{1}$ (Porto Alegre) & $13^{\circ}$ \\
\hline $2^{a}$ (Porto Alegre) & $11^{9}$ \\
\hline $3^{2}$ (Pelotas) & $15^{\circ}$ \\
\hline $4^{2}$ (Santa Maria) & $9^{\mathrm{o}}$ \\
\hline $5^{a}$ (Caxias do Sul) & $3^{\circ}$ \\
\hline $6^{2}$ (Passo Fundo) & $7^{\circ}$ \\
\hline $7^{\text {a }}$ (Bagé) & $14^{9}$ \\
\hline $8^{2}$ (Cachoeira do Sul) & $10^{\circ}$ \\
\hline $9^{a}$ (Cruz Alta) & $5^{9}$ \\
\hline $10^{2}$ (Alegrete) & $16^{2}$ \\
\hline $11^{\text {(Erechim) }}$ & $6^{\circ}$ \\
\hline $12^{\mathrm{a}}$ (Santo Ângelo) & $8^{0}$ \\
\hline $13^{a}$ (Santa Cruz do Sul) & $12^{\circ}$ \\
\hline $14^{\mathrm{Q}}$ (Santa Rosa) & $2^{9}$ \\
\hline $15^{a}$ (Palmeira das Missōes) & $4^{9}$ \\
\hline $16^{2}$ (Lajeado) & 12 \\
\hline
\end{tabular}

de mortalidade infantil $(-0,54)$ e com o baixo peso ao nascer $(-0,41)$. Isto indica que regiões com menor cobertura apresentam piores indicadores de saúde, o que está de acordo com o marco conceitual.

Em termos estatísticos, apenas as correlações entre mortalidade infantil e baixo peso, e entre aquela variável e mortalidade proporcional, atingiram o nível de significância. No entanto, as demais associações, particulatmente aquelas de 0,4 ou mais, não podem ser desprezadas porque o reduzido tamanho amostral (apenas 16 DRSs) limita a probabilidade de atingir resultados significativos.

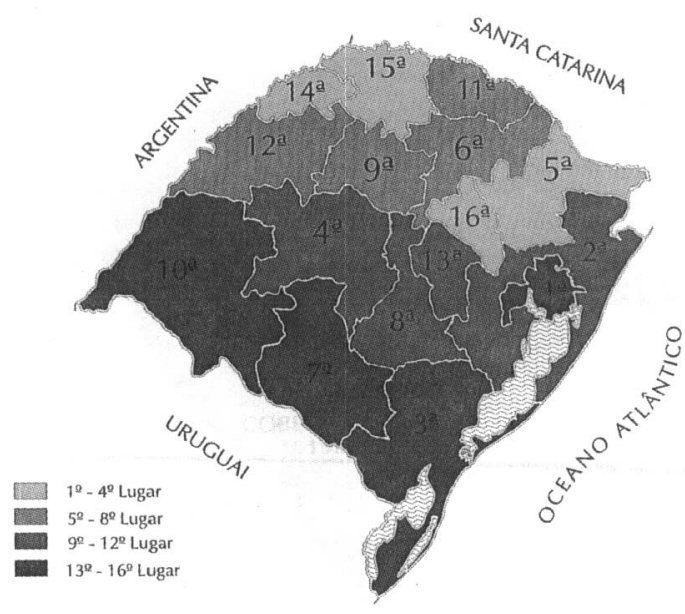

Figura 12. Ordenação das regiōes em termos de indicadores de saúde. Rio Grande do Sul. Divisão em Delegacias Regionais de Saúde. 


\section{Priorização das Regiōes}

Para priorizar as regiões em termos de precariedade dos indicadores de saúde estudados, o seguinte procedimento foi adotado:

a) Os indicadores foram listados em ordem crescente de mortalidade e de frequiência de baixo peso, e a cada DRS foi alocado um valor correspondente a sua posição nesta listagem. Por exemplo, para a variável coeficiente de mortalidade infantil o valor 1 foi dado a Palmeira das Missões (primeiro lugar) e o valor 16 a Alegrete (décimo sexto lugar). A variável cobertura vacinal, por coerência, foi listada em ordem decrescente, com a melhor cobertura recebendo o primeiro lugar e a pior o décimo sexto.

b) Os quatro escores assim obtidos foram somados e as DRSs ordenadas novamente, cabendo portanto o primeiro lugar àquela com as melhores condições de saúde (isto é, com a menor soma de escores), e o último lugar àquela com as piores condições.

c) Aplicados estes critérios, a $4^{\mathrm{a}}, 8^{\mathrm{a}}$ e $12^{\mathrm{a}}$ Delegacias ficaram empatadas em oitavo lugar, enquanto que a terceira e sétima empataram em décimo quarto lugar. Utilizou-se como critério de desempate o coeficiente de mortalidade infantil.

Os resultados obtidos estão listados na Tabela 3 e na Figura 12. As DRSs do norte do Estado (Lajeado, Santa Rosa, Caxias do Sul e Palmeira das Missões) obtiveram as primeiras colocaões, enquanto que os últimos lugares foram obtidos por DRSs do sul (Bagé, Pelotas e Alegrete). Estes dados podem ser de grande utilidade para a priorização de intervenções visando a melhoria da saúde e dos cuidados infantis.

\section{Discussão}

A análise de tendências temporais dos indicadores da saúde evidenciou, na década de 1980 , uma redução de cerca de $50 \%$ nos coeficientes de mortalidade de menores de um ano e de crianças de um a quatro anos, assim como na proporção de óbitos de menores de um ano em relação ao total de óbitos. Por outro lado, observou-se as prevalências de baixo peso ao nascer permanecerem estáveis, e houve marcadas flutuações nos níveis de cobertura vacinal.

Chama a atenção a notável queda na mortalidade infantil, a qual não foi acompanhada de melhoria no peso ao nascer. Isto parece indicar que esta redução tenha sido devida basicamente a intervenções médicas pontuais, como o controle das doenças infecciosas e o atendimento ao parto.
Por outro lado, as prevalências de baixo peso ao nascer, ao permanecerem elevadas, sugerem que não houve alterações nas condições subjacentes de saúde e nutrição maternas. Infelizmente, não existem dados comparáveis sobre as prevalências de desnutrição infantil que permitam traçar uma série temporal e contribuir para o melhor entendimento dessas tendências.

Por outro lado, as marcantes flutuações nos níveis de cobertura vacinal mostram que não houve, durante a década, investimento constante na área preventiva. Maiores coberturas foram a 1 cançadas em determinados anos através de campanhas específicas, e não de uma melhoria sustentável nos serviços básicos de saúde.

Mesmo assim, para os três indicadores citados, o Rio Grande do Sul alcançava, em 1990, lugar de destaque na conjuntura nacional, sendo superior à quase totalidade dos demais Estados. No entanto, se comparado com os níveis de outros países latino-americanos como Cuba, Chile, Uruguai e Costa Rica, ou ainda com os países industrializados, os dados ainda deixam bastante a desejar.

A análise geográfica dos indicadores de mortalidade e de baixo peso ao nascer mostrou uma distribuição desigual dentro do Estado, sendo mais precárias as condições de saúde nas regiões Sul e Oeste, além da Região Metropolitana. O Norte do Estado, por outro lado, mostrou as melhores condições. Estas desigualdades regionais reproduzem os resultados de estudos realizados há mais de uma década, os quais mostraram forte associação entre a estrutura fundiária e a saúde das crianças. Como naquela época, as regiões caracterizadas pelas grandes propriedades de terra apresentavam as piores condições sanitárias ${ }^{8}$.

O estudo geográfico da cobertura vacinal, por outro lado, foi menos elucidativo do que o dos demais indicadores, em parte porque altas coberturas podem ser obtidas através de esforços localizados, não apresentando uma determinação social e econômica tão marcada quanto os indicadores de níveis de saúde. Mesmo assim, pode ser também observada tendência a coberturas mais baixas no Sul do que no Norte do Estado.

Finalmente, criou-se uma pontuação de forma a identificar as regiões do Estado com maiores necessidades na área da saúde infantil. O esquema proposto de ordenação das regiões com um escore simplificado, separando as "piores" das "melhores", não visa substituir, em termos de pesquisa acadêmica, técnicas mais sofisticadas como análise de conglomerados. No entanto, este enfoque permite uma análise simples, passível de ser 
realizada em nível de serviços de saúde, que permite identificar áreas prioritárias para intervenções. Esta análise mostrou que toda a fronteira Sul do Estado, assim como a Região Metropolitana, apresentaram as condições mais precárias. Os níveis de saúde e assistência melhoraram marcadamente no sentido do Norte do Estado. Estas informações podem ser de grande utilidade para a priorização das intervenções nas áreas médica e social.

VICTORA, C.G. et al. [The health situation of children in area of southern Brazil, 1980-1992: tendencies over time and geographical distribution]. Rev. Satide Pública, 28: 423-32, 1994. Although vital statistics are of paramount importance for health planning and program evaluation, few Brazilian states have vital registration systems with either sufficient coverage or agility to achieve these goals. The present analyses, based on data from the state of Rio Grande do Sul, describes time trends and the geographical distribution of infant and child health indicators, including infant mortality rates, proportionate infant mortality, low birthweight and vaccine coverage. From 1980 to 1992, marked reductions were observed for the infant mortality rate (from 39.0 to 19.3 per thousand) and in proportionate infant mortality (from $13.9 \%$ to $5.9 \%$ of all deaths). On the other hand, the prevalence of low birthweight remained stable between 8 and $10 \%$, with a slight increase up to 1991 . DPT vaccine coverage oscillated from year to year, ranging from 79 to $99 \%$. There was clos geographical correlation between the indices of low birthweight and infant mortality in the 17 health districts. The four indicators were combined into a single score for the purpose of identifying those health districts with the greater nedd for intervention. The southern districts, characterized by large land holdings presented the worst health indicators.
Keywords: Infant mortality, trends. Infant, low birth weight. Vital registration.

\section{Referências Bibliográficas}

1. GRANT, J. Situação mundial da infância 1993. Brasília, Unicef, 1992

2. MONTEIRO, C.A.; PINO, H. P.; BENICIO, M.H.A.; VICTORA, C.G. Better prospects for child survival. World Health Forum, 10:222-7, 1989.

3. MONTEIRO, C, A. O mapa da pobreza no Brasil. Brasília, INAN, 1991.

4. MONTEIRO, C.A.; BENIClO, M.H.A.; GOUVElA, N.C. Saúde e nutrição das crianças brasileiras no final da década de 1980. Brasília, INAN, 1991.

5. RIO GRANDE DO SUL (Estado). Secretaria da Saúde e do Meio Ambiente. Estatísticas de saúde: mortalidade 1990. Porto Alegre, 1992. v.16.

6. VICTORA, C.G. Mortalidade infantil, desnutricão e condiçōes socioeconômicas: os casos de São Paulo e do Ceará. In: Congresso Brasileiro de Epidemiologia, $2^{\circ}$ Belo Horizonte, 1992.

7. VICTORA, C.G.; BARROS, F.C.; VAUGHAN, J.P. Epidemiologia da desigualdade: um estudo longitudinal de 6000 crianças brasileiras. $2^{2}$ ed. São Paulo, Hucitec, 1982.

8. VICTORA, C.G. \& VAUGHAN, J.P. Propriedade da terra e saúde infantil no Rio Grande do Sul: as relações entre produção agrícola, desnutrição e mortalidade. Rev. Bras. Estudos Pop., 4:127-51, 1987.

9. WORLD HEALTH ORGANIZATION. The incidence of low birthweight: a critical review of available information. World Health Stat.Q., 33:197-224, 1980.

Recebido para publicação em 8.2.1994 Aprovado para publicação em 11.8.1994 